\title{
IMPLEMENTAÇÃO DE UM MODELO COM O USO DE CFD PARA A OBTENÇÃO DE UMA CORRELAÇÃO DO NÚMERO DE NUSSELT EM TANQUES DE MISTURA COM SERPENTINAS HELICOIDAIS
}

\author{
R. JAIMES e J. R. NUNHEZ \\ Universidade Estadual de Campinas, Faculdade de Engenharia Química \\ E-mail para contato: jaimes@feq.unicamp.br
}

\begin{abstract}
RESUMO - Tanques de mistura com aquecimento são utilizados em diversos processos químicos. Quando reações endotérmicas ou exotérmicas com uma alta liberação ou absorção de energia ocorrem nestes equipamentos, camisas e serpentinas helicoidais ou axiais são empregadas ao mesmo tempo para promover uma maior superfície de transferência de calor. O maior problema associado às serpentinas helicoidais é que estas podem limitar o fluxo no interior do reator, pois as serpentinas fornecem resistência à circulação do fluido. $\mathrm{O}$ objetivo deste trabalho foi desenvolver um modelo 3D com o uso da fluidodinâmica computacional para simular a troca térmica dentro destes equipamentos. Resultados iniciais indicam que o modelo em CFD reproduz a correlação de Nusselt obtida experimentalmente.
\end{abstract}

\section{INTRODUÇÃO}

Reatores ou tanques agitados (em inglês STR - Stirred Tank Reactors) são amplamente utilizados nas indústrias químicas. As operações de cristalização, extração liquido-liquido, lixiviação, reações catalíticas heterogêneas, fermentação são alguns exemplos de operações industriais realizadas em tanques de mistura. A finalidade principal destes equipamentos consiste em obter uma melhoria na homogeneização, na transferência de calor e massa, além do aspecto reacional (Paul et al., 2004; Joaquim et al., 2007). Para garantir a homogeinização, um o mais impelidores (comumente chamado de agitadores) são utilizados para gerar o fluxo e a mistura desejada.

$\mathrm{O}$ reator tanque agitado consiste normalmente em um tanque cilíndrico equipado com um impelidor acoplado a um motor. O desempenho destes equipamentos é afetado pela localização dos internos e ou pelo modo de operação. Em reações com troca térmica, onde o calor de reação é alto, é necessário um dispositivo para aquecimento ou esfriamento do sistema e garantir a segurança de operação. Para manter o fluido no reator na temperatura desejada em reações com elevados calores de reação, o calor deve ser adicionado o removido normalmente por serpentinas internas (axial ou helicoidal) para promover a transferência de calor.

Estudos experimentais têm a grande vantagem de tratar com a configuração real, contudo é normalmente um processo caro e consome um tempo demasiadamente grande. Além disso, a experimentação muitas vezes não pode ser realizada por questões de segurança ou pela dificuldade 
de reprodução das condições reais. Como alternativa tem-se a aplicação da dinâmica dos fluidos computacional (CFD) que tem por característica apresentar um relativo baixo custo e rapidez, além de fazer as investigações experimentais mais eficientes, possibilitando um entendimento mais profundo dos processos de escoamento. Nesse sentido, este trabalho consiste em desenvolver um modelo com o uso de CDF para a obtenção da correlação do Número de Nússelt em reatores de tanque agitado com serpentinas helicoidais. Este trabalho é uma continuação da pesquisa de Pedrosa e Nunhez (2003). A simulação foi validada comparando os resultados do coeficiente de transferência de calor na parede da serpentina com dados obtidos experimentalmente no trabalho de Oldshue e Gretton (1954). Além disso, foi estudado o comportamento do coeficiente de transferência de calor com o aumento de velocidade de rotação do impelidor (tipo Rushton de seis pás).

\section{MATERIAIS E MÉTODOS}

A seguir é descrita a técnica numérica empregada na simulação e na análise da influência da posição das serpentinas de um reator de tanque agitado, em relação ao escoamento do fluido e à distribuição da temperatura em regime turbulento.

\subsection{Abordagem Matemática do Fenômeno Físico}

O fenômeno físico envolvido no movimento do fluido no processo de agitação e mistura foi modelado com o uso das equações de conservação de massa, momentum e energia. A seguir serão apresentadas as equações que governam a movimentação de um fluido em qualquer escoamento.

Conservação de massa:

$$
\frac{\partial \rho}{\partial t}+\rho\left[\frac{\partial v_{i}}{\partial x_{i}}+\frac{\partial v_{j}}{\partial x_{j}}+\frac{\partial v_{k}}{\partial x_{k}}\right]=0
$$

Conservação de momentum:

$$
\rho\left[\frac{\partial v_{i}}{\partial t}+\frac{\partial\left(v_{i} \cdot v_{j}\right)}{\partial x_{j}}\right]=-\frac{\partial p}{\partial x_{i}}+\frac{\partial}{\partial x_{j}}\left[\mu\left(\frac{\partial v_{i}}{\partial x_{j}}+\frac{\partial v_{j}}{\partial x_{i}}-\frac{2}{3} \delta_{i j} \nabla \cdot \vec{v}\right)\right]+\rho g_{i}+\sum F_{i}
$$

Conservação de energia:

$$
\frac{\partial(\rho E)}{\partial t}+\frac{\partial\left[v_{i}(\rho E+p)\right]}{\partial x_{i}}=\frac{\partial}{\partial x_{i}}\left[k_{e f} \frac{\partial T}{\partial x_{i}}-\sum_{j \prime} h_{j^{\prime}} J_{j^{\prime}, i}+v_{j}\left(\tau_{i j}\right)_{e f}\right]+S_{j i}
$$

Foi utilizado o modelo de turbulência SST (Shear Stress Transport), que é composto pelos modelos de turbulência k- $\omega$ e k- $\varepsilon$. 


\subsection{Caso de Estudo}

A configuração geométrica do reator consiste de um tanque cilíndrico de fundo reto com quatro chicanas localizadas perpendicularmente à parede do tanque, um conjunto de serpentinas distribuídas ao longo de seu comprimento e equipado com um impelidor tipo Rushton de seis pás. $\mathrm{O}$ dimensionamento do tanque agitado com serpentinas helicoidais foi baseado nas dimensões do equipamento usado no trabalho experimental de Oldshue e Gretton (1954). Água a $25{ }^{\circ} \mathrm{C}$ (densidade $=1000 \mathrm{~kg} / \mathrm{m} 3$, viscosidade dinâmica $=0,001 \mathrm{~kg} / \mathrm{ms}$ ) foi usada como fluido de estudo A Figura 1 mostra o equipamento experimental utilizado e na Tabela 1 as dimensões geométricas.

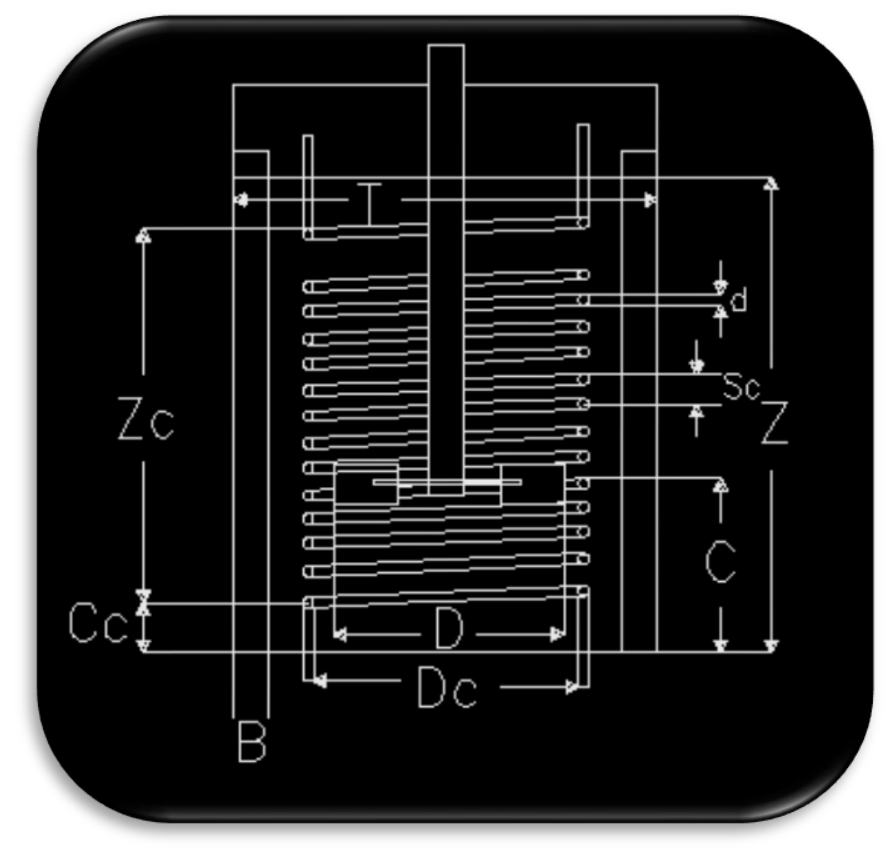

Figura 1 - Geometria experimental usada por Oldshue e Gretton (1954).

Tabela 1 - Dimensões do tanque simulado

\begin{tabular}{lcc}
\multicolumn{1}{c}{ Dimensão } & Símbolo & Valor $(\mathrm{m})$ \\
\hline Diâmetro do tanque & $T$ & 1,22 \\
Altura de liquido & $Z$ & 1,22 \\
Altura do impelidor & $C$ & 0,41 \\
Diâmetro do impelidor & $D$ & 0,41 \\
Espessura do disco & $s$ & 0,01 \\
Comprimento da pá & $D_{b}$ & 0,10 \\
Altura da pá & $D_{w}$ & 0,08 \\
Diâmetro da hélice & $D_{c}$ & 0,87 \\
Diâmetro de tubo & $d$ & 0,04 \\
Separação entre tubos & $S_{c}$ & 0,09
\end{tabular}




\subsection{Simulação Numérica}

A criação da geometria foi feita utilizando a ferramenta CAD (DesignModeler) disponível pelo software ANSYS CFX 14.0. Para reduzir o custo computacional, o domínio que contém o fluido no tanque agitado foi dividido em duas partes simétricas, abrangendo duas chicanas e três pás do impelidor cada. Somente uma foi modelada. Na parte simulada, o domínio na simulação é composto de duas regiões: uma região interna chamada rotativa e uma região externa chamada estacionária. A região estacionária é constituída pelas paredes, serpentinas e chicanas do tanque enquanto a região rotativa é conformada pelo domínio que compõe o impelidor. Uma malha foi criada para discretizar o domínio em pequenos volumes de controle, onde as equações de conservação são aproximadas por equações algébricas. Para a criação desta malha foi utilizado o software ANSYS CFX meshing. A Figura 2 mostra as malhas não estruturadas tridimensionais com elementos tetraédricos e prismáticos nos domínios rotativo e estacionário.

(a)
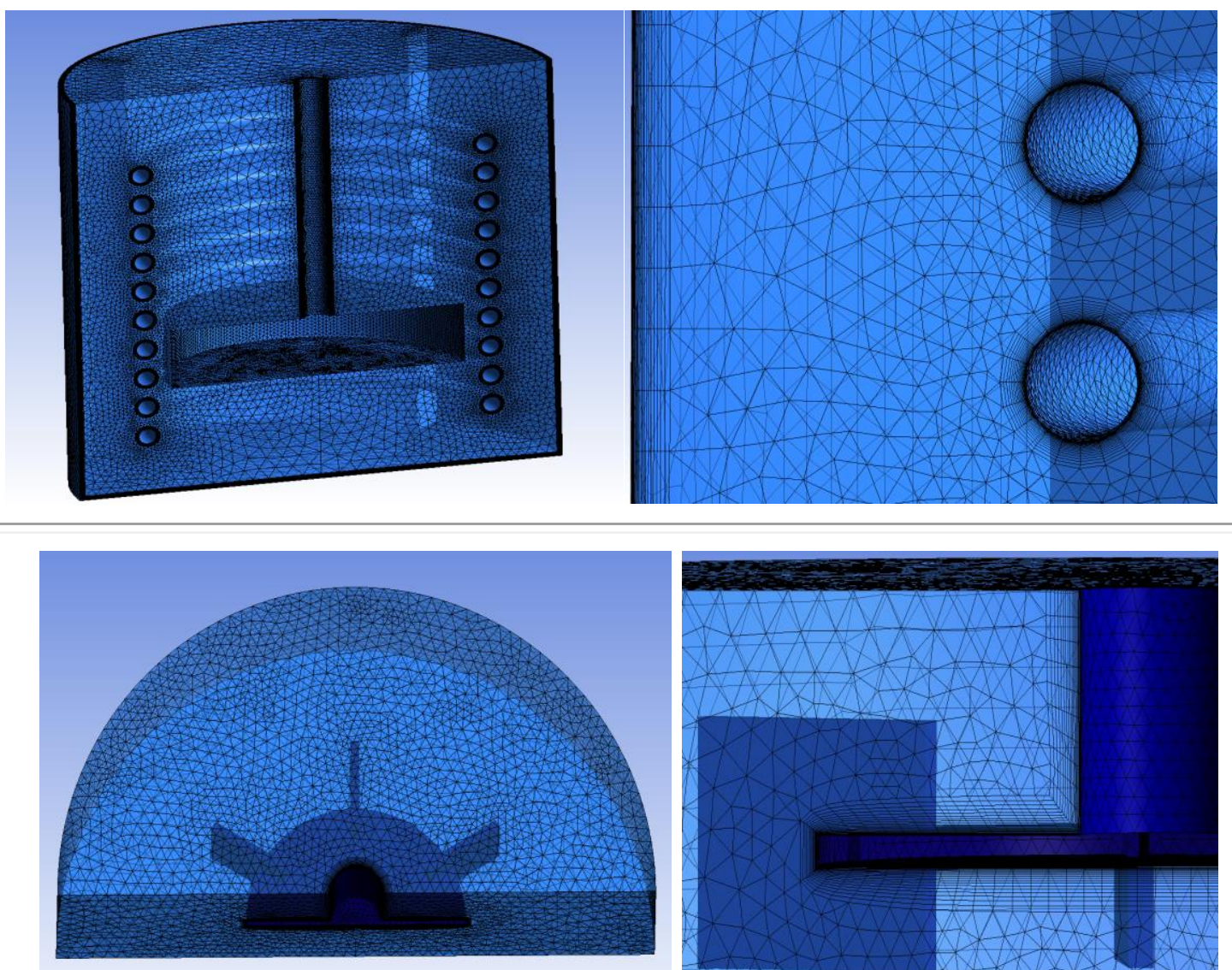

(b)

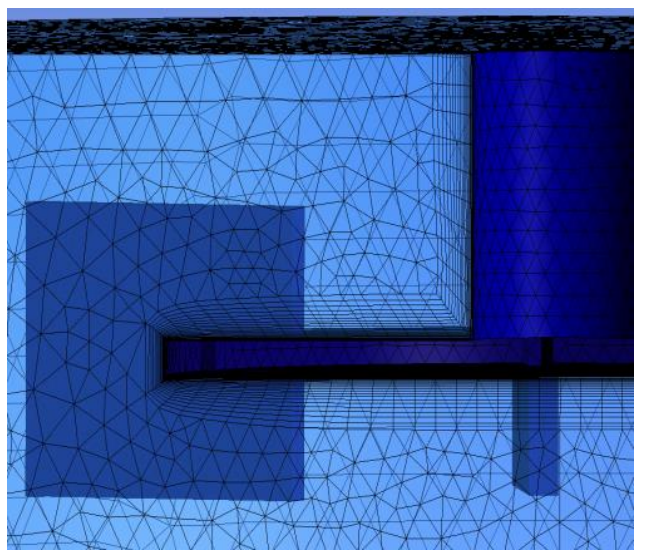

Figura 2 - Malha não estruturada 3D com elementos tetraédricos e prismáticos. (a) Malha superficial do domínio estacionário. (b) Malha superficial do domínio rotativo. 
Nas regiões normais às superfícies como paredes, chicanas, serpentinas, impelidor e eixo foram aplicados elementos prismáticos. Foi usada uma distância adimensional na faixa de $0,1<\mathrm{y}^{+}$ $<1$ com 20 prismas dentro da camada limite com o intuito de garantir bons resultados pelo modelo de turbulência, o cálculo do escoamento cerca da superfície da parede (Spogis e Nunhez, 2009). Nas simulações foram utilizados 370000 elementos no domínio rotativo e 4500000 no domínio estacionário. Previamente foi feito uma análise de estabilidade da malha, criando diferentes densidades de malha até que os resultados numéricos mostraram independência da discretização. As seguintes condições de contorno e suposições foram adotadas para as simulações em regime totalmente transiente.

Paredes e fundo do tanque: Foi utilizada uma condição de não escorregamento, logo a velocidade do fluido é reduzida nas regiões perto da parede ate que o fluido em contato atinja a velocidade nula na parede.

Chicanas: Não existe deslizamento de líquido, logo a velocidade é nula na parede.

Superfície livre: Na superfície livre do líquido, as tensões de cisalhamento e velocidade axial são nulas e uma superfície plana é assumida. Por tanto, pode ser aplicada na simulação a condição de livre escorregamento na superfície superior do tanque.

Impelidor e eixo-árvore: É assumido que a velocidade do fluido na parede assume a mesma velocidade angular definida pela rotação do impelidor. Por tanto será considerada parede de não escorregamento.

Tubos da serpentina: Não existe deslizamento de líquido, logo a velocidade é nula na parede. Será assumido que há suficiente líquido frio entrando nas serpentinas para manter a temperatura na parede constante de $5{ }^{\circ} \mathrm{C}$. Na condição de temperatura fixa na parede o fluxo de calor da parede ao fluido é calculado como:

$$
q=k_{f}\left(\frac{\partial T}{\partial n}\right)_{\text {parede }}=h_{f}\left(T_{w}-T_{f}\right)
$$

Em que $h_{f}$ é o coeficiente de transferência de calor, $T_{w}$ é a temperatura na parede da serpentine e $T_{f}$ é a temperatura no seio do fluido. O modelo de turbulência SST (Shear Stress Transport) foi escolhido devido a sua abordagem mista entre os modelos $k-\omega$ e $k-\varepsilon$, a qual oferece bons resultados na estimativa das variáveis de interesse perto da parede e no seio do fluido. A abordagem de rotação MFR-Frozen rotor é utilizada para predizer o escoamento em regime transiente. Uma periodicidade de $180^{\circ}$ é usada para reduzir o tempo computacional requerido. $\mathrm{O}$ passo do tempo e o intervalo da simulação foram definidos utilizando estratégias para modelar a interação chicana-impelidor encontradas na literatura (Lane et al., 2000; Murthy e Joshi, 2008). As simulações foram realizadas com um passo de tempo de $0,02 \mathrm{~s}$ e o intervalo de tempo da simulação foi aquele tempo no qual o coeficiente de transferência de calor fosse constante $(8 \mathrm{~s})$. Na literatura existem muitas diferenças nestes tempos de simulação, geralmente encontrando-se em uma faixa de tempo desde 10 ate 44 voltas do impelidor (Lane et al., 2000; Murthy e Joshi, 2008). Neste caso as simulações foram feitas em regime transiente com número aproximado de 40 voltas do impelidor, considerando valores RMS residuais inferiores a $1 \times 10^{-5}$ 
para garantir a convergência dos resultados.

\section{RESULTADOS}

Com o intuído de validar o modelo proposto para um tanque de mistura com serpentinas helicoidais, a Figura 3 apresenta a comparação entre o coeficiente de transferência de calor (número de Nusselt) gerado pelos resultados da simulação e o coeficiente de transferência de calor calculado através da correlação de Nusselt obtida no trabalho experimental de Oldshue e Gretton (1954). Na Figura 3 foi observado que para as cinco velocidades de rotação analisadas (200, 250, 300, 350, e 400 rpm) o número de Nusselt calculado no modelo CFD apresenta boa concordância com os dados experimentais obtidos através da correlação de Oldshue e Gretton (1954).

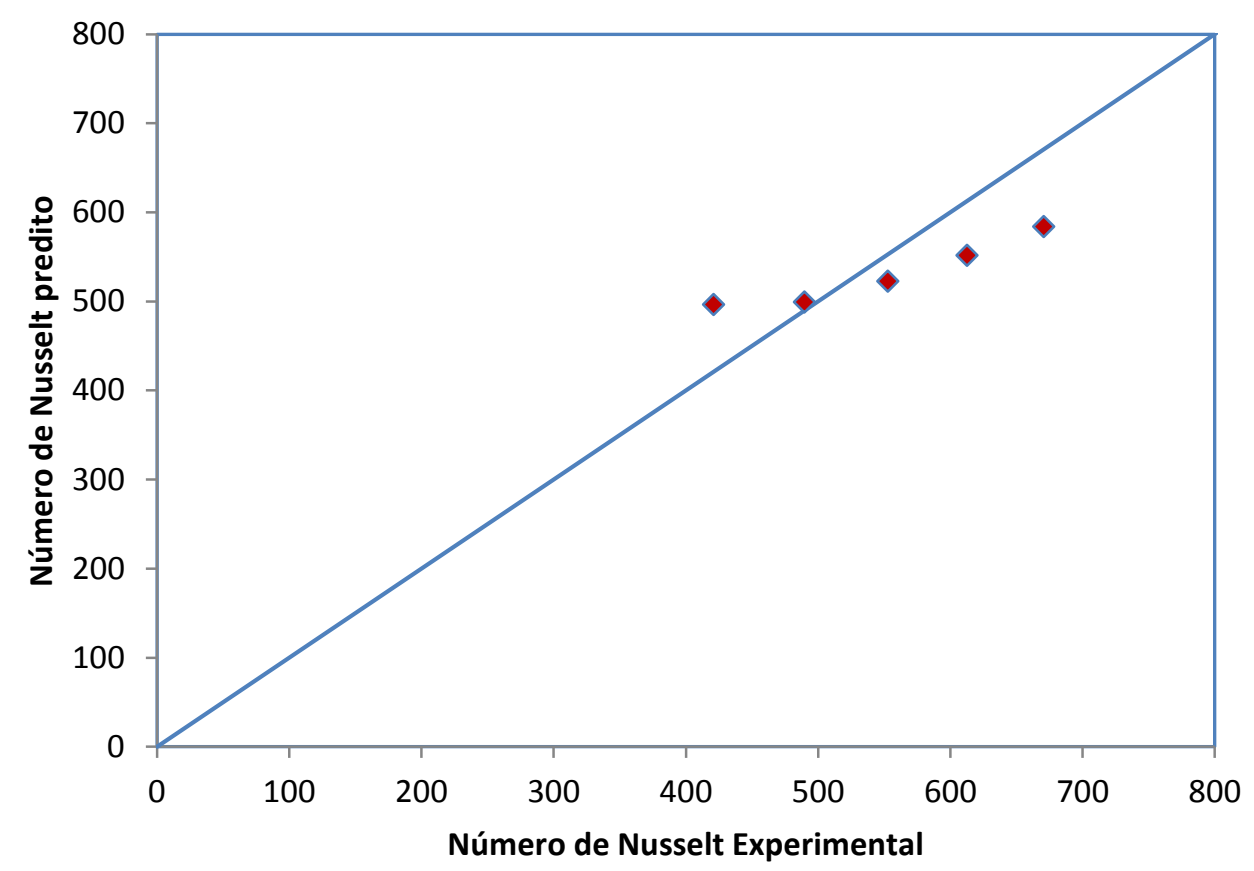

Figura 3 - Comparação do número de Nusselt experimental e do número de Nusselt calculado pelo modelo CFD.

Os gráficos de vectores preditos para os planos radial e axial são mostrados na Figura 4. Na Figura 4 (b) pode-se observar que a rotação do impelidor produz um fluxo intenso na direção radial o qual, ao bater com a parede, divide a região em duas zonas de recirculação distintas, acima e abaixo do impelidor, o que é típico neste tipo de impelidores. Além disso, pode ser observado na Figura 4 (a) que a velocidade média do fluido diminui quando se encontra com a serpentina fazendo com que a circulação do fluido seja dificultada.

O efeito da velocidade do impelidor (número de Reynolds) na transferência de calor (número de Nusselt) sobre as condições apresentadas na Tabela 1 é mostrado na Figura 5. Na figura pode ser observado que o coeficiente de transferência de calor aumenta com o incremento da velocidade de rotação do impelidor, devido ao fato que para altos valores da velocidade existe maior intensidade da mistura que por sua vez gera uma alta turbulência no meio, fazendo mais efetiva a troca térmica do sistema. 

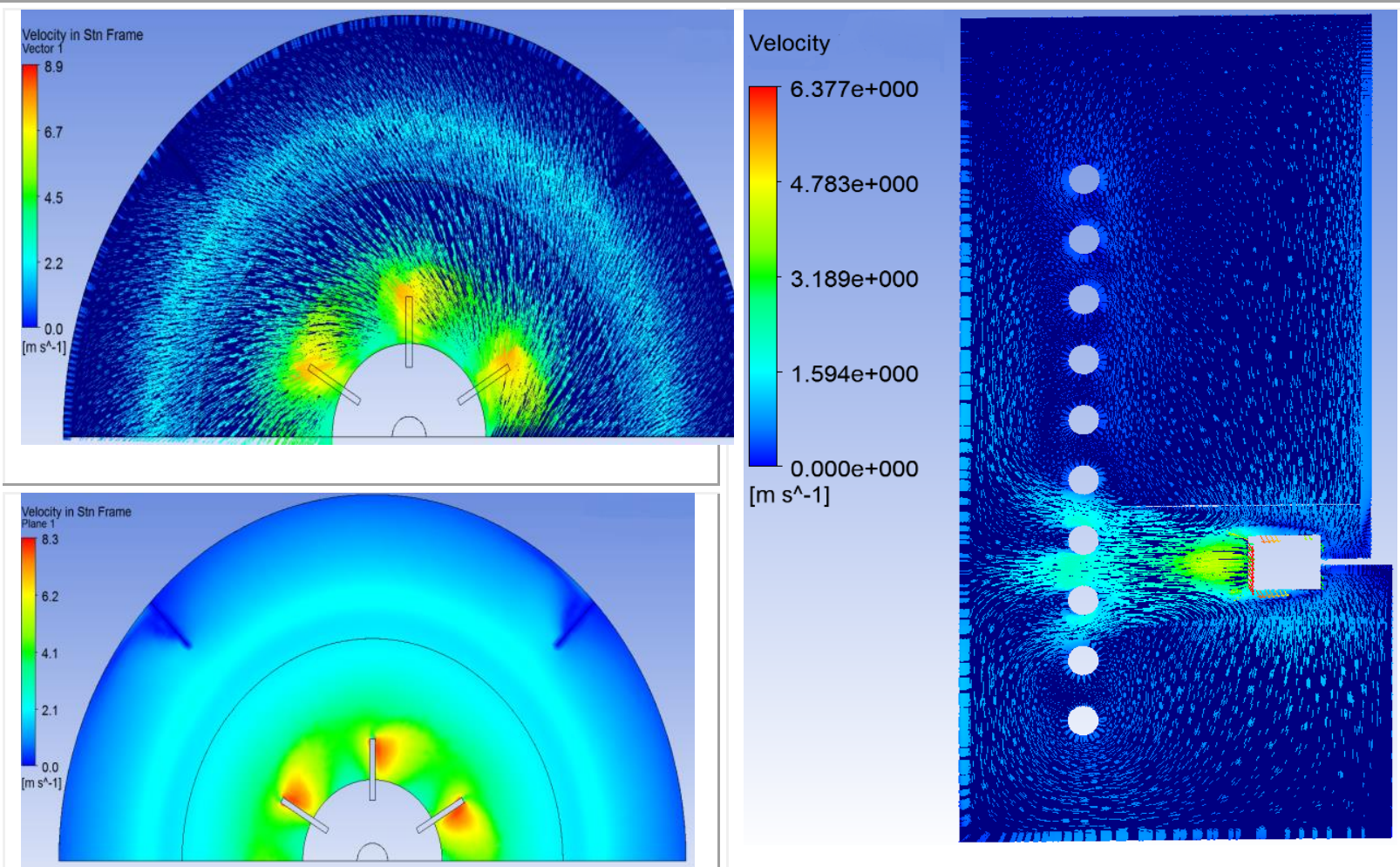

(a)

(b)

Figura 4 - Campo vectorial da velocidade predita $\mathrm{R}_{\mathrm{e}}=8,2 \times 10^{5}$. (a) Plano radial na altura do disco. (b) Plano axial na direção da pá.

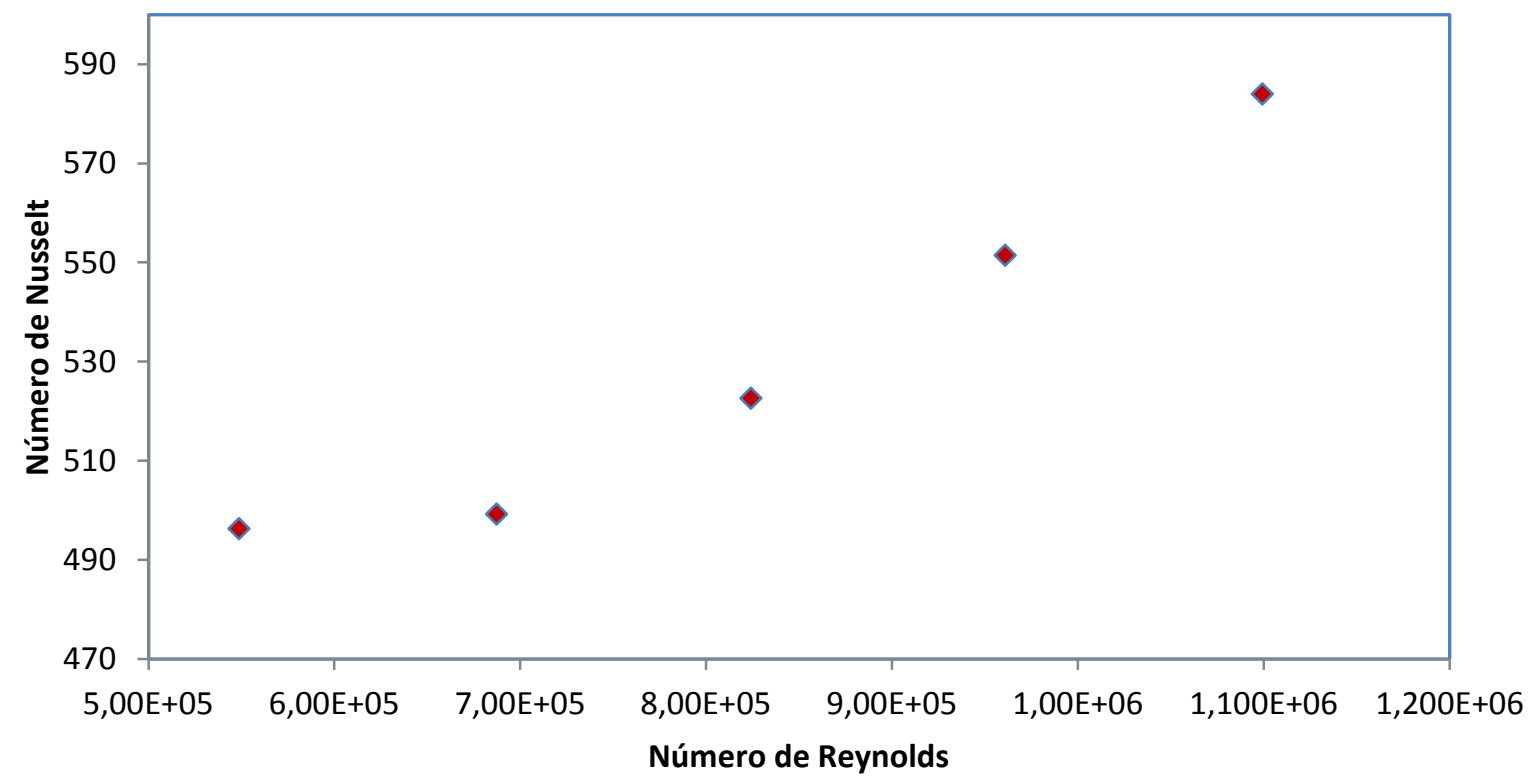

Figura 5 - Efeito da velocidade de rotação na transferência de calor para o impelidor tipo Rushton. 


\section{CONCLUSÕES}

A predição do modelo CFD tem sido comparada com os dados experimentais de Olshue e Gretton (1954) para quatro velocidades de rotação. O número de Nusselt obtido no modelo foi comparado com o valor predito pela correlação experimental apresentado uma variação de $15 \%$. O modelo tridimensional mostrou boa concordância na predição qualitativa como quantitativa em termos da obtenção do número de Nusselt e do campo de fluxo característico em impelidores radiais (Rushton de seis pás). Além disso, foi estudado o efeito da velocidade de agitação no coeficiente de transferência de calor, encontrando-se um aumento no coeficiente de transferência de calor com o aumento da velocidade do impelidor, como esperado.

\section{REFERENCIAS}

JOAQUIM, C.; CEKINSKI, E.; NUNHEZ, J.; URENHA, L. Agitação e mistura na indústria. Rio de Janeiro: Editora Livros Técnicos e Científicos LTC, 2007.

LANE, G.; SCHWARZ, M.; EVANS, G. Comparison of CFD methods for modelling of stirred tanks. Akker, H. E. A. V. D. \& Derksen, J. J. Elsevier Science, Amsterdam, 2009.

MURTHY, B.; JOSHI, J. Assessment of standard, RSM and LES turbulence models in a baffled stirred vessel agitated by various impeller designs. Chem. Eng. S., v. 63, p. 5468-5495, 2008.

OLDSHUE, J.; GRETTON, A. Helical coil heat transfer in mixing vessels. Chem. Eng. P., v. 50, p. 615-621, 1954.

PAUL, E.; ATIEMO-OBENG, V.; KRESTA, S. Handbook of industrial mixin: Science and Practice. São Paulo: Wiley-Interscience, 2004.

PEDROSA, S.; NUNHEZ, J. Improving heat transfer in stirred tanks cooled by helical coils, Brazilian J. Chem. Eng., v. 20, n. 2, p. 111-120, 2003.

SPOGIS, N. and NUNHEZ, J. Design of a high-efficiency hydrofoil through the use of computational fluid dynamics and multiobjective optimization, AIChE J., v. 55, n. 7, p. 17231735, 2009. 